\title{
Effect of A Priori Information in Pixel Domain Wyner-Ziv Video Coding
}

\author{
Ralph Hänsel, Erika Müller \\ Institute of Communications Engineering \\ University of Rostock \\ Rostock, Germany \\ \{ralph.haensel,erika.mueller\}@uni-rostock.de
}

\begin{abstract}
Distributed video coding (DVC) is an interesting research topic for emerging application scenarios requiring low complexity video encoding. The central Slepian-Wolf (SW) coding, in a DVC system, exploits the cross correlation between the side information and the original frame. But, it does not exploit the source entropy of the input symbols, which is exploited by entropy coding in conventional video transmission systems.

It is shown, that the $\mathrm{SW}$ coding performance is significantly reduced in case of reduced source entropy (non-uniformly distributed symbols). We propose two algorithms, which adjust the SW coding to the source entropy. Thus, the SW coding performance is increased by $16 \%$ and the overall rate distortion performance, of the proposed DVC system, is increased by up to $0.82 \mathrm{~dB}$. Furthermore, the low encoding complexity is preserved.
\end{abstract}

Keywords-Distributed Source Coding (DSC), Distributed Video Coding (DVC)

\section{INTRODUCTION}

Modern multimedia communication systems demand efficient video coding algorithm. State-of-the-art video coding (e.g. MPEG-4, H.264/AVC, H.264/SVC) are very well suited for broadcasting scenarios, wheres the complex video encoding is done only ones, but the decoding is done many times at the client side. That approach is less adequate for emerging application scenarios, requiring low encoder complexity.

In contrast to conventional coding systems, distributed video coding (DVC) gives the ability to develop low complexity encoders. It is based on the theories of D. Slepian and J. Wolf [1] as well as A. D. Wyner and J. Ziv [2], which proof that a distributed coding system can reach the same performance as a conventional coding system.

Distributed video coding is also well suited for errorrobust transmission and multiview video coding. Further application fields and perspectives are given in [3], [4].

One of the major technologies in a distributed video coding system is the Slepian-Wolf (SW) coding. It exploits the correlation between the side information $Y$ and the original frame $X$. The pixel values of the original frame are non-uniformly distributed, thus, the source entropy is reduced. In conventional video coding the source entropy is

978-1-4244-7286-4/10/\$26.00 (C)2010 IEEE.

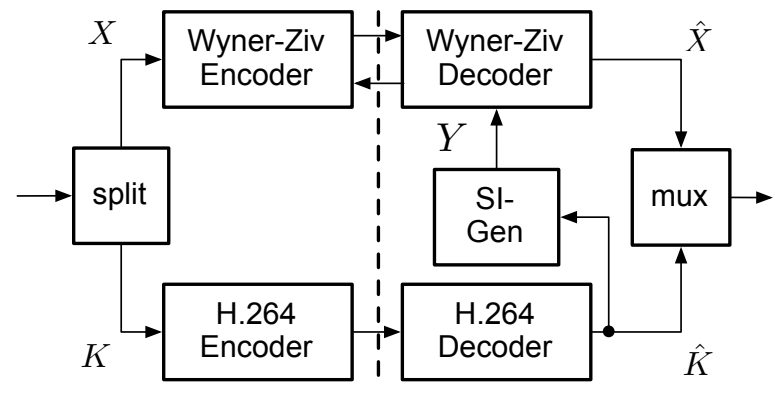

Figure 1. Pixel domain DVC coding scheme

exploited by the entropy coding. Whereas, the SW coding in DVC does not exploit the source entropy by default. Furthermore, the SW coding performance is significantly reduced in case of reduced source entropy, which is shown in section IV.

We propose two methods to adjust the SW coding to the reduced source entropy. Simulations show that the performance of SW coding is significantly increased, if the SW coding is adjusted to non-uniformly distributed symbols (sec. IV). An overview of the related work is given in sec. III. Furthermore, we propose a priori information transmission (AIT, sec. V-A) and a priori information estimation (AIE, sec. V-A) algorithm for our pixel domain DVC system (sec. II). Simulations show that the overall rate distortion performance is increased by up to $0.8 \mathrm{~dB}$ if AIT or AIE is applied (sec. VI). At the end conclusions and remarks on further research are given in section VII.

\section{System SETUP}

Our DVC coding scheme (fig. 1) splits up the input sequence into Key $K$ and Wyner-Ziv $X$ frames. On the one hand, the Key frames are encoded and decoded by the H.264/AVC intra (JM12.4 [5]) codec. On the other hand, the WZ frames are encoded by the low complexity WZ encoder. The corresponding decoding is performed by the WZ decoder, where an estimate of the original frame is available (side information $Y$ ). The side information $Y$ is estimated by temporal interpolation between the adjacent Key frames.

The WZ encoding includes quantization of the WZ frame pixel values by a $2^{M}$-step linear quantizer. The obtained 
quantization symbols $q$ are split up into $M$ bit planes $q^{(b)}$. Subsequently the binary SW encoder generates parity bits (turbo code, [6]), which are transmitted to the decoder on request.

In the first step, the decoder generates the side information $Y$ by temporal interpolation. The temporal interpolation is performed by the BiMESS (Bidirectional Motion Estimation and Spatial Smoothing, [7]) or by the PBTI (Pixel Based Temporal Interpolation, [8]) algorithm. Decoding in the WZ decoder includes SW decoding based on the side information $Y$ (estimate of $X$ ) and requested parity bits from the encoder. SW decoding is very much like turbo decoding, it only works if a specific number of parity bits is available. Therefore more and more parity bits are requested until SW decoding is successful. The decoded quantization symbols $\hat{q}$ are used in the reconstruction process to form the decoded frame $\hat{X}$. Based on the discussed reconstruction algorithms in [9], we choose the side information clipping reconstruction algorithm for our DVC system. At least the decoder stores the decoded frames in the output sequence.

The overall performance of the DVC system is significantly influenced by the quality of the side information and by the performance of the SW coding.

\section{RELATED WORK}

Slepian-Wolf coding for nonuniform sources was first studied in [10]. The Slepian-Wolf coder was implemented by a Turbo Coding (TC) scheme. The decoder was adjusted to the source statistic by supplying a priori information. In [10] the SW coding scheme was not applied to a practical distributed video coding system, thus the problem of providing the source statistic at the decoder was not studied.

Furthermore, Distributed Arithmetic coding (DAC) based Slepian-Wolf coding for nonuniform sources was studied in [11]. It was shown, that the TC scheme performs better for low correlation between the sources $X$ and $Y$, whereas the DAC performs better for for high correlation. Furthermore the DAC is not so much affected by the nonuniform distribution of source $X$. At least [12] studied nonuniform sources for LDPC based SW coders.

\section{A PRIORI INFORMATION IN SW CODING}

The pixel values in a video frame are typically nonuniformly distributed. Due to $2^{M}$-step linear quantization the quantization symbols $q$ and its bit planes $q^{(b)}$ are also non-uniformly distributed. Therefore the entropy $H(q)$ is not at a maximum $\left(H(q)<M, H\left(q^{(b)}\right)<1\right)$. Figure 2 shows the entropy of each bit plane for the sequences Coastguard, Foreman and Soccer. The source entropy of the bit planes one and two of the sequence Foreman and of all three planes of Soccer is significantly reduced.

The binary simmetric channel (BSC) model is used for performance evaluation of the SW coding scheme. The

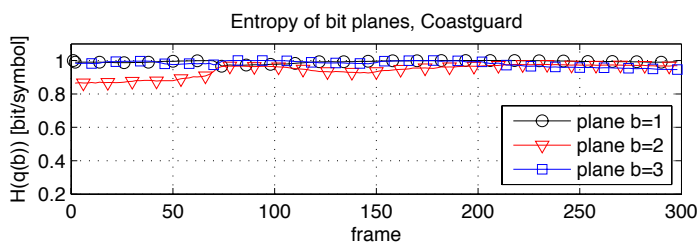

(a) Coastguard

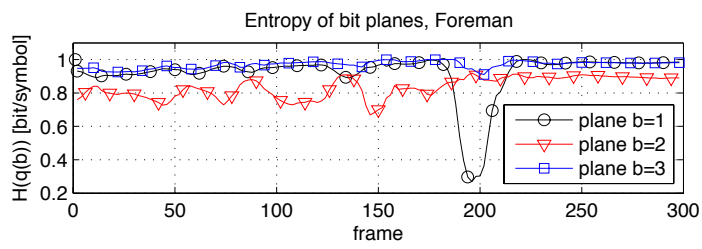

(b) Foreman

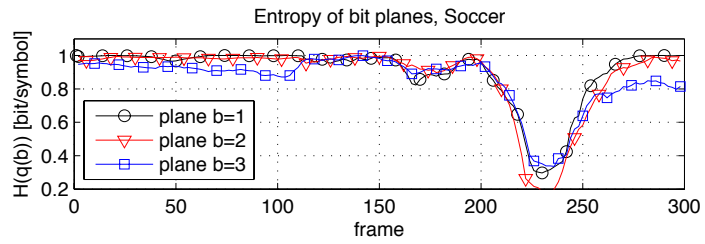

(c) Soccer

Figure 2. Entropy of each quantization symbols bit plane $q^{(b)}$

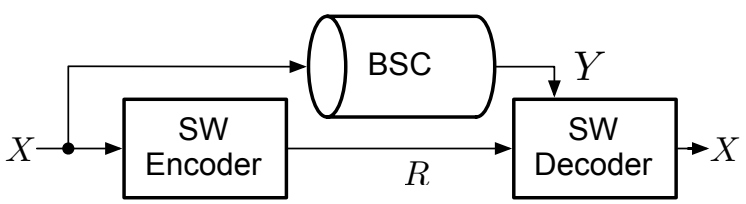

Figure 3. Setup for SW channel simulation - a priori information not incorporated

channel is characterized by the cross over probability $e$. Furthermore, the channel capacity $C$ depends on the crossover probability and is given in eq. 1 .

$$
\begin{aligned}
C & =1+(1-e) \log _{2}(1-e)+e \log _{2}(e) \\
& =1-H(Y \mid X)
\end{aligned}
$$

For uniform input symbols $x_{i}$ the conditional entropy $H(X \mid Y)$ is equal to $H(Y \mid X)$. For non uniform sources $H(X \mid Y)$ highly depends on the entropy of $X$. Equations 3 and 4 are used to calculate the conditional entropy for SW evaluation on nonuniform sources.

$$
\begin{aligned}
H(X \mid Y) & =H(X)+H(Y \mid X)-H(Y) \\
& =-\sum_{i} \mathrm{P}\left(x_{i}\right) \sum_{j} \mathrm{P}\left(y_{j} \mid x_{i}\right) \log _{2} \mathrm{P}\left(y_{j} \mid x_{i}\right)(4)
\end{aligned}
$$

\section{A. Non a priori information usage}

The SW coding performance is evaluated by the simulation setup shown in figure IV-A, where the correlation 


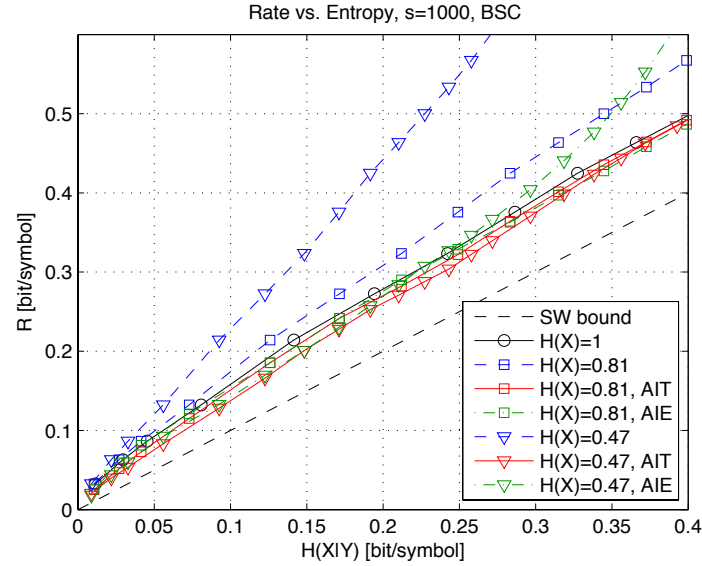

(a) absolute rate

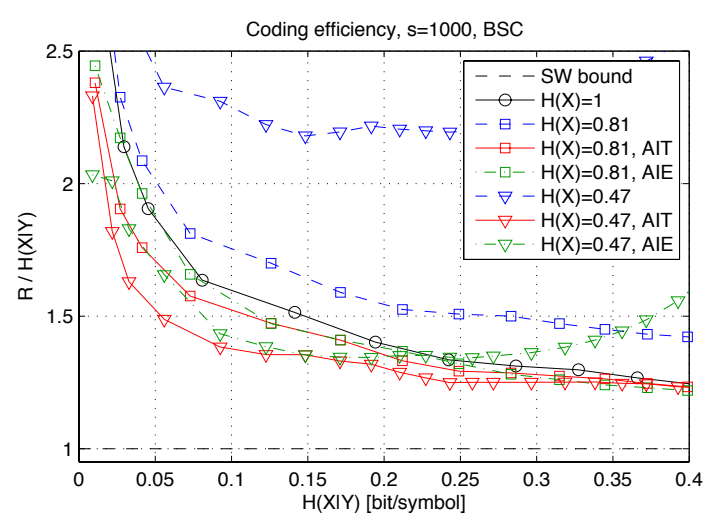

(b) rate normalized to the conditional entropy

Figure 4. SW coding performance, blocklength $s=1000 \mathrm{bit}$

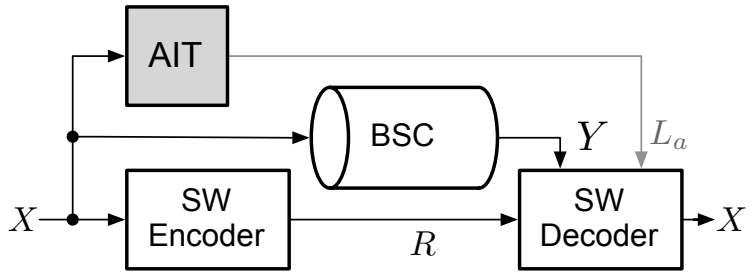

Figure 5. Setup for SW channel simulation - a priori information incorporated at the encoder (AIT)

$H(X \mid Y)$ between the original information $X$ and the side information $Y$ is modeled by a binary symmetric channel (BSC).Figure 4 shows, that the SW coding performance is significantly reduced if the source entropy $H(X)$ is not maximum (blue dashed curves). The gap to the SW bound is larger compared to the case of maximum source entropy (black solid curve).

\section{B. A Priori Information Transfer (AIT)}

The SW decoder can incorporate a priori information (eq. 5), which indicates the non-uniform distribution of $X$. We propose to calculate the a priori information at the

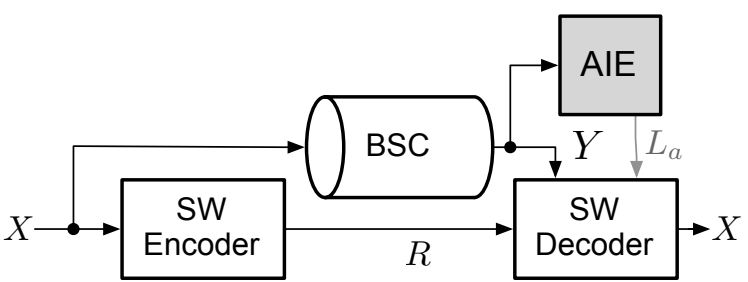

Figure 6. Setup for SW channel simulation - a priori information incorporated at the decoder (AIE)

encoder and the transmission to the decoder for improved SW coding performance (fig. IV-B). The a priori information transfer (AIT) algorithm shows good coding performance close to the SW bound. For a practical source entropy of $H(X)=0.81$ (Foreman, 2nd bit plane, fig. 2(b)) the coding performance is increased by $16 \%$ compared to the default SW coding without AIT.

$$
L_{a, A I T}=\ln \frac{\mathrm{P}(X=0)}{\mathrm{P}(X=1)}
$$

\section{A Priori Information Estimation (AIE)}

The a priori information is calculated at the encoder for the AIT algorithm. Furthermore, it needs to be transmitted to the decoder. Thus, a transmission overhead is added to the system. The distribution of $X$ and the distribution of the side information $Y$ is highly correlated. Thus, the a priori information can also be estimated at the decoder side base on the side information (fig. IV-C), avoiding the transmission overhead of AIT. Therefore, secondly, we proposed the a priori information estimation (AIE) algorithm based on the side information $Y$. The a priori information is estimated at the decoder side (eq. 6). Simulations show that the performance of AIE (green, dash-dot line, fig. 4) is close to the performance of AIT, for high correlation between $X$ and $Y$. Hence, AIE is an alternative to AIT, with the advantage of not introducing a transmission overhead.

$$
L_{a, A I E}=\ln \frac{\mathrm{P}(Y=0)}{\mathrm{P}(Y=1)} \approx \ln \frac{\mathrm{P}(X=0)}{\mathrm{P}(X=1)}
$$

It was shown, that a reduced source entropy has significant impact to the SW coding performance. We propose to adjust the SW decoder to a reduced source entropy by AIT and AIE, with high SW coding performance close to the SW bound.

\section{Proposed Wyner-Ziv Video Coding}

In section IV the impact of reduced source entropy to the SW coding performance was discussed. Furthermore, two algorithm were proposed to adapt the SW coding to the source entropy for increased performance. In this section the proposed algorithms are adapted to our pixel domain DVC system (sec. II). 


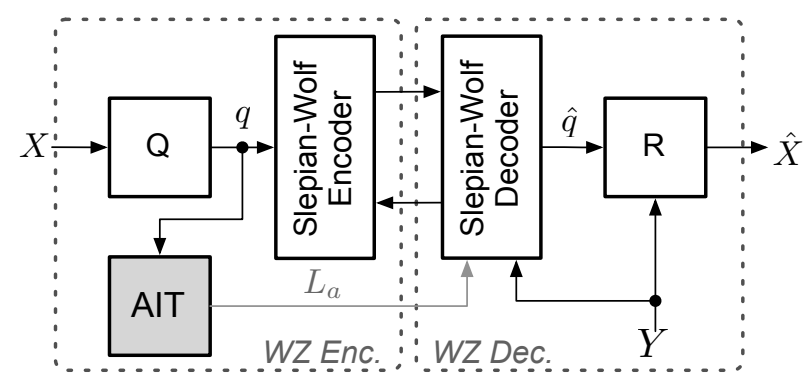

Figure 7. Proposed DVC system with AIT

\section{A. A Priori Information Transfer (AIT)}

A priori information transfer (AIT) is included in our pixel domain DVC system as shown in figure 7. After the uniform quantization, the statistic of the quantization symbol bit planes is analyzed in the AIT process. Furthermore, the a priori information for each bit plane $L_{a, A I T}\left(\hat{q}_{i}^{(b)}\right)$ is calculated and transmitted to the decoder. Each LLR value (Log Likelihood Ratio, $L_{a, A I T}$ ) is quantized to an 8 bit value prior transmission. Therefore a transmission overhead of $M \times 8$ bit is added by AIT per frame (e.g. $0.36 \mathrm{kbps} @$ $30 \mathrm{fps}, M=3, G O P=2$ ).

The SW decoder incorporates the a priori information ( $L_{a}$, eq. 7$)$ in conjunction with the intrinsic information $\left(L_{c}\right.$, eq. 8) extracted from the side information $y$ and extrinsic information $L_{e}$ gathered from the transmitted parity bits to form the a posteriori information (decoding result, $L$, eq. 9).

$$
\begin{aligned}
& L_{a, A I T}\left(\hat{q}_{i}^{(b)}\right)=\ln \frac{\mathrm{P}\left(q_{i}^{(b)}=0\right)}{\mathrm{P}\left(q_{i}^{(b)}=1\right)} \\
& L_{c}\left(\hat{q}_{i}^{(b)}\right)=\ln \frac{\mathrm{P}\left(y_{i} \mid q_{i}^{(b)}=0\right)}{\mathrm{P}\left(y_{i} \mid q_{i}^{(b)}=1\right)} \\
& L\left(\hat{q}_{i}^{(b)}\right)=L_{c}\left(\hat{q}_{i}^{(b)}\right)+L_{a, A I T}\left(\hat{q}_{i}^{(b)}\right)+L_{e}\left(\hat{q}_{i}^{(b)}\right)
\end{aligned}
$$

As shown in section IV the performance is increased by adjusting the SW coding to the source entropy. The impact to the overall RD performance of the DVC system is discussed in conjunction with the simulation results in section VI.

\section{B. A Priori Information Estimation (AIE)}

The SW coding can be adjusted to the source entropy by AIT, where a priori information needs to be transmitted to the decoder. An alternative is the AIE algorithm, where the a priori information is estimated at the decoder side. In section IV it was shown that AIE has good performance close to AIT, if the side information $Y$ and the original symbol $X$ is highly correlated.

The AIE algorithm is implemented in our pixel domain DVC system as shown in figure 8 . The encoder is not modified, thus, remaining at very low computational complexity.

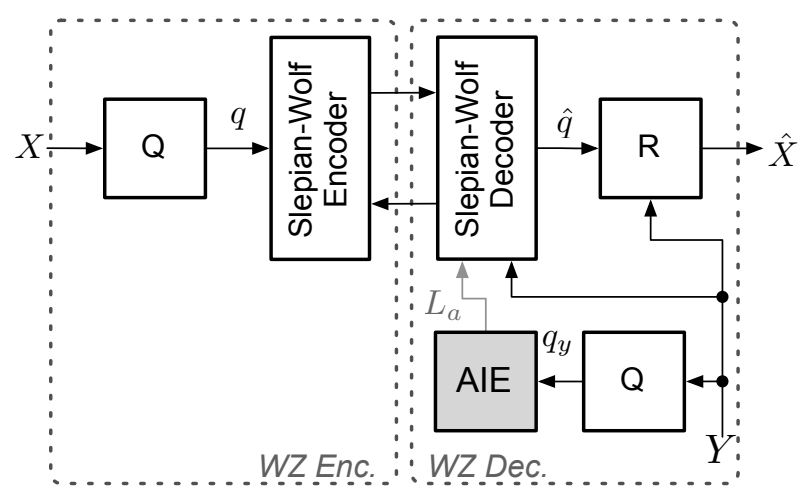

Figure 8. Proposed DVC system with AIE

An additional quantization block is added to the decoder to obtain the quantization symbols $q_{y}$ based on the side information $Y$. They are used as an estimate of the original quantization symbols $q$ to calculate the a priori information $L_{a, A I E}\left(\hat{q}_{i}^{(b)}\right)$. The a priori LLR is calculated by equation 10 and incorporated in the SW decoding algorithm (eq. 11).

$$
\begin{gathered}
L_{a, A I E}\left(\hat{q}_{i}^{(b)}\right)=\ln \frac{\mathrm{P}\left(q_{y, i}^{(b)}=0\right)}{\mathrm{P}\left(q_{y, i}^{(b)}=1\right)} \\
L\left(\hat{q}_{i}^{(b)}\right)=L_{c}\left(\hat{q}_{i}^{(b)}\right)+L_{a, A I E}\left(\hat{q}_{i}^{(b)}\right)+L_{e}\left(\hat{q}_{i}^{(b)}\right)
\end{gathered}
$$

\section{Simulation Results}

The proposed algorithms were evaluated for the QCIF sequences Coastguard, Foreman and Soccer at $30 \mathrm{fps}$. The overall RD (rate distortion) performance is shown in the figures 9, 10 and 11. Simulation results are carried out for the BiMESS [7] and PBTI [8] side information generation algorithm. Furthermore, the quantization parameter $M=1$ and $M=2$ were used. A KWK GOP (group of picture) structure was used. The key $(\mathrm{K})$ frames were coded by the H.264/AVC reference codec (JM12.4, [5]) and the WynerZiv (W) frames were processed by the proposed DVC system (sec. V).

\section{A. A Priori Information Transfer (AIT)}

The overall performance of the proposed DVC system applying AIT for the sequence Coastguard is not significantly improved compared to the basic DVC system. Due to the high source entropy of the quantization symbol bit planes (fig. 2(a)) the extracted a priori information value $L_{a}$ is very small, causing similar RD performance. Furthermore, the introduced rate overhead is insignificant compared to the data rate transmitted by the SW encoder. Thus, the $\mathrm{RD}$ performance is not significantly influenced. Table I shows the RD performance gain of the proposed algorithms compared to the basic DVC system (sec. II). For the sequence Coastguard the performance gain for the quantization 

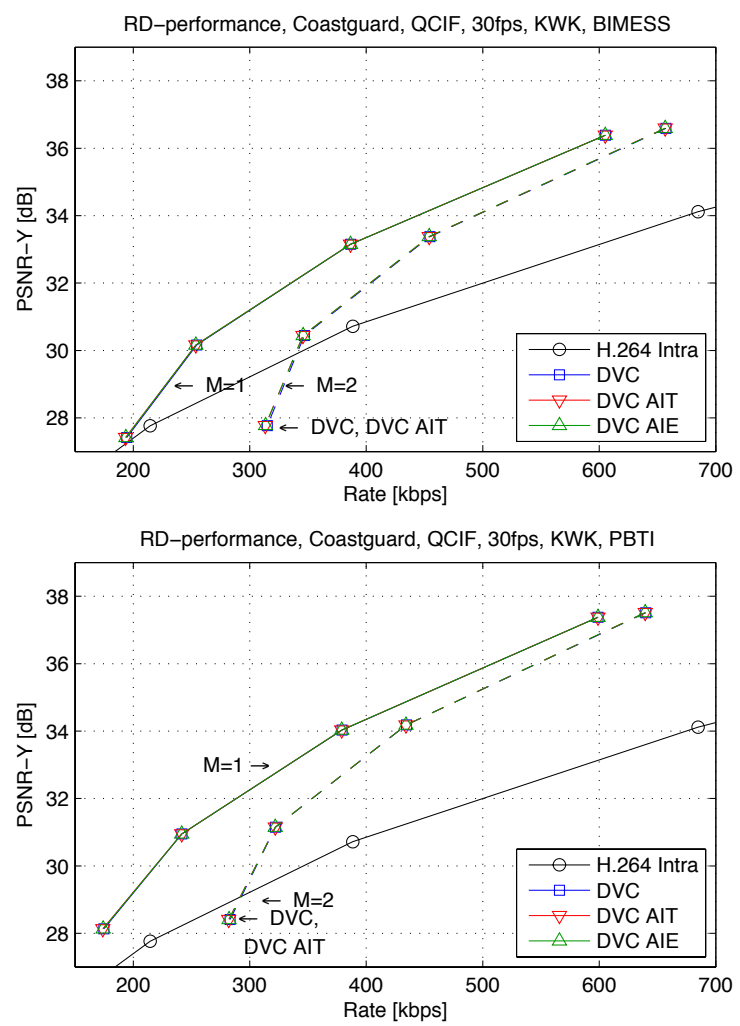

Figure 9. Overall RD performance of WZ coding, Coastguard

parameter $M=2$ is higher than for $M=1$, because the source entropy of the second bit plane is lower.

For the sequence Foreman, the RD performance is improved by up to $0.82 \mathrm{~dB}$ (tab. I). Furthermore, the highest gain is accomplished for the second bit plane, because the average source entropy of the 2nd bit plane is very low $\left.\overline{H\left(q^{(2)}\right)} \approx 0.8 \mathrm{bit} / \mathrm{symbol}\right)$. Furthermore, the RD performance is improved for all evaluated coding conditions.

High RD performance gains are also shown for the Soccer sequence (fig. 11, tab. I) for all quantization parameter. Because, the source entropy for all bit planes of the quantization symbols is low (frames $210 \ldots 250$, fig. 2(c)).

Table I

MAX RD PERFORMANCE GAIN FOR THE PROPOSED AIT (A PRIORI INFORMATION TRANSFER) AND AIE (A PRIORI INFORMATION ESTIMATION)

\begin{tabular}{|c|c|c|c|}
\hline & Coastguard & Foreman & Soccer \\
\hline \multicolumn{4}{|l|}{ BiMESS [7] } \\
\hline$M=1$ & $0.05 \mathrm{~dB}$ & $0.01 \mathrm{~dB}$ & $0.53 \mathrm{~dB}$ \\
\hline$M=2$ & $0.15 \mathrm{~dB}$ & $0.82 \mathrm{~dB}$ & $0.74 \mathrm{~dB}$ \\
\hline$M=1$ & $0.03 \mathrm{~dB}$ & $0.01 \mathrm{~dB}$ & $0.43 \mathrm{~dB}$ \\
\hline$M=2$ & $0.14 \mathrm{~dB}$ & $0.73 \mathrm{~dB}$ & $0.60 \mathrm{~dB}$ \\
\hline \multicolumn{4}{|l|}{$\begin{array}{l}\text { PBTI [8] } \\
\end{array}$} \\
\hline$M=1$ & $0.05 \mathrm{~dB}$ & $0.00 \mathrm{~dB}$ & $0.20 \mathrm{~dB}$ \\
\hline$M=2$ & $0.08 \mathrm{~dB}$ & $0.52 \mathrm{~dB}$ & $0.33 \mathrm{~dB}$ \\
\hline$M=1$ & $0.03 \mathrm{~dB}$ & $0.00 \mathrm{~dB}$ & $0.19 \mathrm{~dB}$ \\
\hline$M=2$ & $0.06 \mathrm{~dB}$ & $0.51 \mathrm{~dB}$ & $0.30 \mathrm{~dB}$ \\
\hline
\end{tabular}
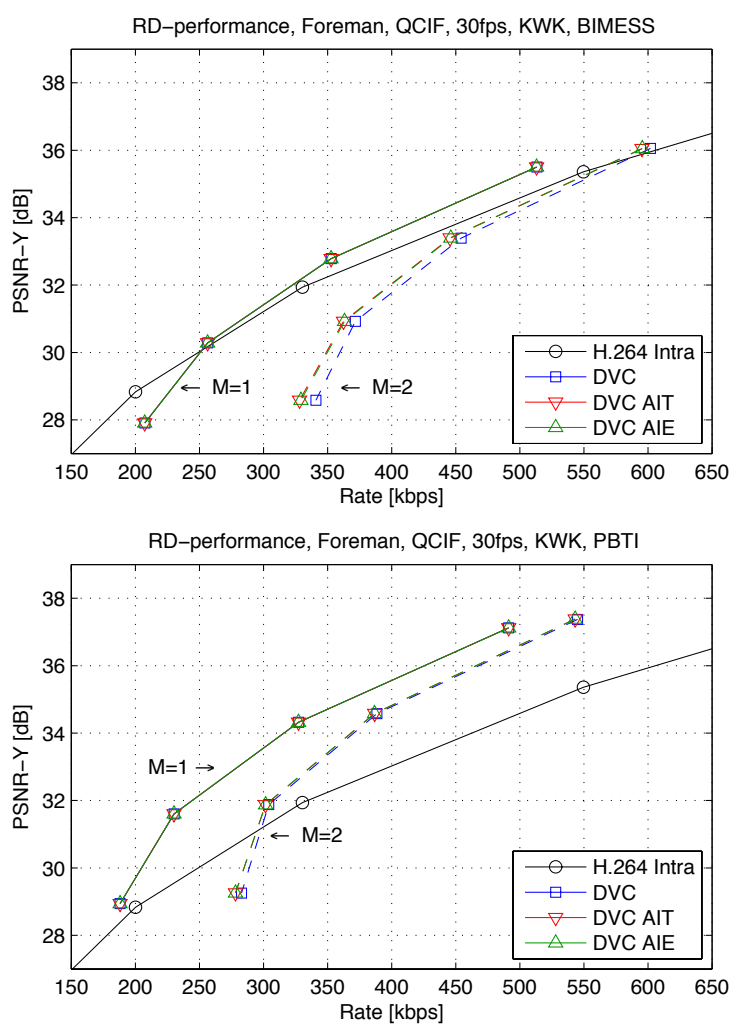

Figure 10. Overall RD performance of $\mathrm{WZ}$ coding, Foreman

Furthermore, the RD performance gain for the DVC system using BiMESS side information generation is greater than the gain for the PBTI based DVC system. The DVC system applying PBTI shows the best overall RD performance. The proposed pixel domain DVC system outperforms the H.264 intra video coding for sequences with medium (Foreman, fig. 10) and high (Coastguard, fig. 9) temporal correlation.

At least the AIT algorithm slightly increases the encoding complexity, because the a priori values need to be calculated at the encoder side.

\section{B. A Priori Information Estimation (AIE)}

In contrast to AIT the AIE algorithm does not add a transmission overhead to the DVC system. The overall RD performance of AIE is close to the performance of AIT (tab. I). Furthermore, the performance is better than the basic DVC system under all evaluated coding conditions.

An advantage of the AIE algorithm compared to the AIT is the slightly lower encoding complexity and no additional transmission overhead. So, the AIE algorithm does not modify the encoder, preserving its low encoding complexity. Furthermore, there is no need to transmit the a priori vales, because the estimation is performed at the decoder side. A brief comparison of both proposed algorithm is given in table II. 

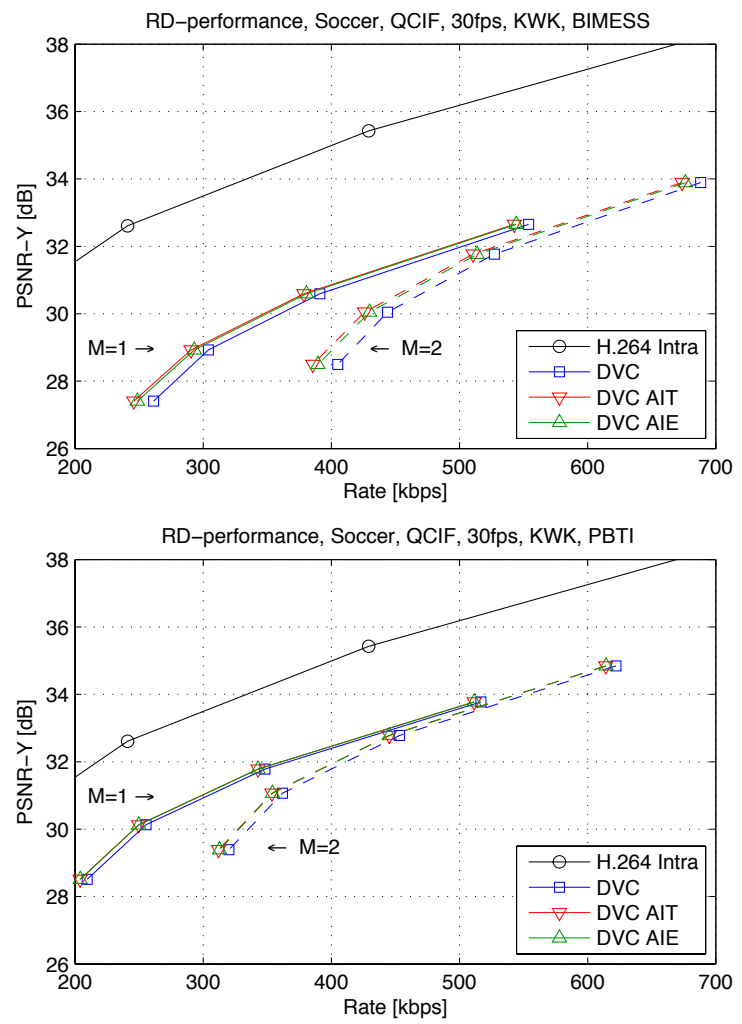

Figure 11. Overall RD performance of WZ coding, Soccer

Table II

COMPARISON OF PROPOSED AIT AND AIE ALGORITHM

\begin{tabular}{|l|l|l|}
\cline { 2 - 3 } \multicolumn{1}{c|}{} & AIT & AIE \\
\hline RD performance & $\begin{array}{l}\text { high RD performan- } \\
\text { ce gain, } \\
\text { up to } 0.82 \mathrm{~dB}\end{array}$ & $\begin{array}{l}\text { moderate RD perfor- } \\
\text { mance gain, } \\
\text { up to 0.74 dB }\end{array}$ \\
\hline $\begin{array}{l}\text { transmission } \\
\text { overhead }\end{array}$ & $\begin{array}{l}M \times 8 \mathrm{bit} / \text { frame, } \\
\text { very low compared } \\
\text { to allover rate } \\
\text { overhead }=0.12 \%\end{array}$ & $\begin{array}{l}\text { 0, estimation per- } \\
\text { formed at the de- } \\
\text { coder }\end{array}$ \\
\hline $\begin{array}{l}\text { computational } \\
\text { encoding complexity }\end{array}$ & slightly increased & $\begin{array}{l}\text { very low, encoder is } \\
\text { not modified }\end{array}$ \\
\hline
\end{tabular}

The choice between AIT and AIE depends on the application case. If it is possible to modify the encoder and add a transmission overhead, it is always the best choice to choose the AIT algorithm.

\section{CONCLUSION}

In this paper, it was shown, that the Slepian-Wolf (SW) coding performance highly depends on the source entropy of the input symbols. The performance is significantly decreased for real world parameters. We proposed two methods, to adapt the SW coding to the source entropy (nonuniformly distributed input symbols). The proposed a priori information transfer (AIT) algorithm performs very close to the Slepian-Wolf bound and increases the SW coding performance by $16 \%$ (bit plane $b=2$, Foreman). In contrast the a priori information estimation (AIE) algorithm shows also good performance if the side information is highly correlated to the original symbols.

Furthermore, we proposed a pixel domain DVC system applying AIT and AIE. Both algorithm improve the overall RD performance by up to $0.82 \mathrm{~dB}(0.74 \mathrm{~dB})$. The AIT algorithm adds a small transmission overhead, which is insignificant compared to the overall rate. Furthermore, the encoding complexity is slightly increased. On the other hand the AIE algorithm does not modify the encoder, thus, neither transmission overhead nor additional encoding complexity is added.

\section{REFERENCES}

[1] D. Slepian and J. Wolf, "Noiseless Coding of Correlated Information Sources," IEEE Trans. on Information Theory, vol. 19, no. 4, pp. 471-480, July 1973.

[2] A. D. Wyner and J. Ziv, "The Rate-Distortion Function for Source Coding with Side Information at the Decoder," IEEE Trans. on Info. Theory, vol. 22, no. 1, pp. 1-10, Jan 1976.

[3] F. Pereira, P. Correia, E. Acosta, L.Torres, C. Guillemot, M. Ouaret, F. Dufaux, T. Ebrahimi, R. Leonardi, M. Dalai, and S. Klomp, "Application scenarios and functionalities for DVC,” DISCOVER DVC, Tech. Rep., 2006.

[4] F. Pereira, L. Torres, C. Guillemot, T. Ebrahimi, R. Leonardi, and S. Klomp, "Distributed Video Coding: Selecting the Most Promising Application Scenarios," Signal Processing: Image Communication, vol. 23, pp. 339-352, 2008.

[5] K. Sühring, "H.264/AVC reference software," http://iphome. hhi.de/suehring/tml/.

[6] D. Rowitch and L. Milstein, "On the performance of hybrid fec/arq systems using rate compatible punctured turbo (rcpt) codes," IEEE Trans. on Comm., vol. 48, no. 6, pp. 948-959, June 2000.

[7] J. Ascenso, C. Brites, and F. Pereira, "Improving Frame Interpolation with Spatial Motion Smoothing for Pixel Domain Distributed Video Coding," in Proc. EURASIP, 2005.

[8] S. Sofke, R. Hänsel, and E. Müller, "Human Visual System aware Decoding Strategies for Distributed Video Coding," in Proc. Picture Coding Symposium PCS, 2009.

[9] R. Hänsel and E. Müller, "Improved Reconstruction for Distributed Video Coding," in Proc. of the International Conference on Ultra Modern Communications (ICUMT), St.Petersburg, Russia, 2009.

[10] J. Li, Z. Tu, and R. Blum, "Slepian-wolf coding for nonuniform sources using turbo codes," in Proceedings. DCC, 2004.

[11] V. Toto-Zarasoa, E. Magli, A. Roumy, and G. Olmo, "On distributed arithmetic codes and syndrome based turbo codes for slepian-wolf coding of non uniform sources," in EURASIP, 2009.

[12] V. Toto-Zarasoa, A. Roumy, and C. Guillemot, "Non-uniform source modeling for distributed video coding," in EUSIPCO, 2010 . 